\title{
Pulmonary Neurofibromatosis with Bilateral Bronchiectasis: A Rare Thoracic Manifestation
}

\author{
Manoj Kumar Pandey, Jyoti Bajpai', Surya Kant', Hemant Kumar, Poornima Mishra² \\ Department of Respiratory Medicine, Ram Manohar Lohiya Institute of Medical Sciences, 'Department of Respiratory Medicine, King George's Medical University, \\ Department of Pathology, T. S. M Medical College and Hospital, Lucknow, Uttar Pradesh, India
}

\section{Abstract}

Neurofibromatosis type 1 (NF1), also known as von Recklinghausen's disease, is an autosomal dominant genetic disorder. The incidence of NF is 1 in 3000 individuals. Approximately one-half of the cases are familial, while the remaining occur sporadically due to germ cell mutations. The clinical manifestation of NF1 may include a café-au-lait macule usually 5-15 mm in diameter, multiple neurofibromas, axillary or inguinal freckling, optic glioma, Lisch nodules, and a distinctive bony lesion. Although very rare, pulmonary manifestation in neurofibromatosis can occur in the form of mediastinal neurofibromas, pleural neurofibromas, tracheobronchial neurofibroma, interstitial lung disease, bullous lung disease, and cystic lung disease. Here, we present a rare case of pulmonary neurofibroma with bronchiectasis.

Keywords: Bronchiectasis, histopathological examination, kyphoscoliosis, neurofibroma

\section{INTRODUCTION}

Neurofibromatosis, which was first described in 1882 by von Recklinghausen, is an autosomal dominant genetic disorder with an incidence of approximately 1 in 2600-3000 individuals. ${ }^{[1]}$ About half of the cases are inherited, while the remaining occur sporadically due to germ cell mutations. Neurofibromatosis type 1 (NF1) affects males and females equally and affects individuals of all racial and ethnic backgrounds.

The clinical manifestation of NF1 may include a café-au-lait macule usually 5-15 mm in diameter, multiple neurofibromas, axillary or inguinal freckling, optic glioma, Lisch nodules, and a distinctive bony lesion. ${ }^{[2]}$ Although very rare, thoracic manifestation in neurofibromatosis can occur in the form of scoliosis, kyphoscoliosis, mediastinal neurofibromas, pleural neurofibroma, interstitial lung disease, cystic lung disease, and bullous lung disease. ${ }^{[3]}$ NF1-related lung disease is a rare but increasingly recognized, high morbidity-associated condition. Scarce data exist regarding the prevalence, clinical characteristics, and pathophysiology of NF1-related pulmonary disease. NF1 may involve lung in the form of bullae, cysts, emphysema, bibasilar reticular opacities, interstitial fibrosis, and/or ground-glass opacities. The relationship between

\begin{tabular}{|l|l|}
\hline \multicolumn{2}{|c|}{ Access this article online } \\
\hline Quick Response Code: & Website: \\
\hline & www.ijrc.in \\
& \\
&
\end{tabular}

smoking and NF1-related lung disease is also unclear. ${ }^{[4]}$ Neurogenic tumor (neurofibroma) of the lung is very rare. Only few cases have been reported in the literature. ${ }^{[5]}$ Here, we present a rare case of bilateral bronchiectasis with an incidental mass in a patient with von Recklinghausen's disease. The mass was later diagnosed to be a neurofibroma on histopathology.

\section{Case Report}

A 45-year-old ex-smoker male presented to our department with the complaints of breathlessness and cough with purulent expectoration for the past 6 months. The patient also had high-grade fever for the past 2 weeks. There was a history of repeated episodes of similar complaints in the past. He was prescribed several courses of antibiotics, resulting in some relief. On examination, he was febrile but hemodynamically stable. He was tachypneic, and his oxygen saturation was

\section{Address for correspondence: Prof. Surya Kant, Department of Respiratory Medicine, King George's Medical University, Lucknow, Uttar Pradesh, India. E-mail: skantpulmed@gmail.com}

This is an open access journal, and articles are distributed under the terms of the Creative Commons Attribution-NonCommercial-ShareAlike 4.0 License, which allows others to remix, tweak, and build upon the work non-commercially, as long as appropriate credit is given and the new creations are licensed under the identical terms.

For reprints contact: WKHLRPMedknow_reprints@wolterskluwer.com

How to cite this article: Pandey MK, Bajpai J, Kant S, Kumar H, Mishra P. Pulmonary neurofibromatosis with bilateral bronchiectasis: A rare thoracic manifestation. Indian J Respir Care 2021;10:132-5.

Received: $14-05-2020$

Accepted: $12-08-2020$ Published: $31-01-2021$ 
$92 \%$ on room air. There were multiple, soft, fleshy, sessile, nontender nodules all over the body surface, diagnosed as neurofibromas [Figure 1]. Kyphoscoliosis and diffuse coarse crackles were present on chest examination. There was absence of clubbing.

His laboratory investigations showed a hemoglobin of $12.1 \mathrm{~g} \%$ and total white cell count of $12,000 / \mathrm{mm}^{3}$ (neutrophils: $68 \%$, lymphocytes: $27 \%$, and eosinophil $5 \%$ ). Total serum IgE was $270 \mathrm{kU} / 1$. Viral markers were negative. No acid-fast bacilli (AFB) were seen on sputum smear. GeneXpert for AFB was negative. Sputum smear for Gram stain was positive for Gram-negative bacilli. Sputum culture was positive for Pseudomonas aeruginosa and sensitive to piperacillin, amikacin, and ciprofloxacin. Spirometry revealed a restrictive pattern (postbronchodilator forced expiratory volume in $1 \mathrm{~s} /$ forced vital capacity [FVC] $81 \%$ predicted and FVC $66 \%$ predicted).

The chest X-ray posteroanterior view showed heterogeneous opacity in the left upper zone and right paracardiac region

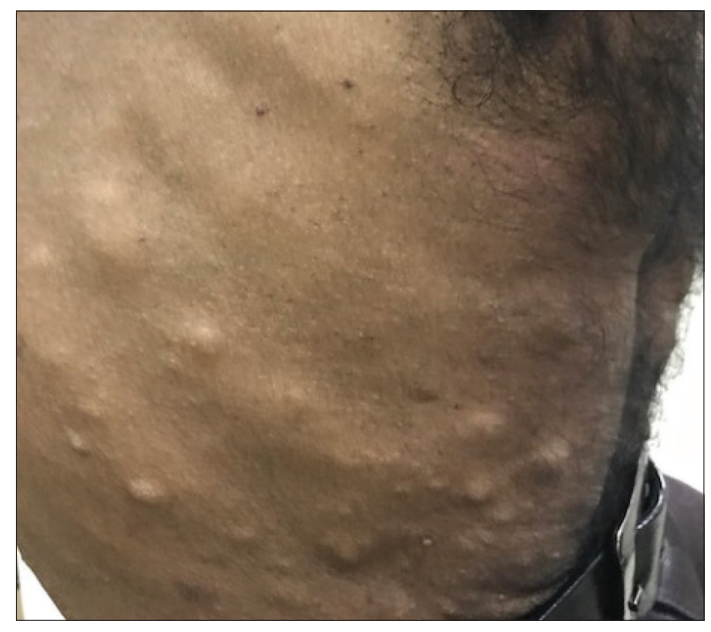

Figure 1: Multiple, soft, fleshy, sessile, nontender nodules over the lateral chest wall

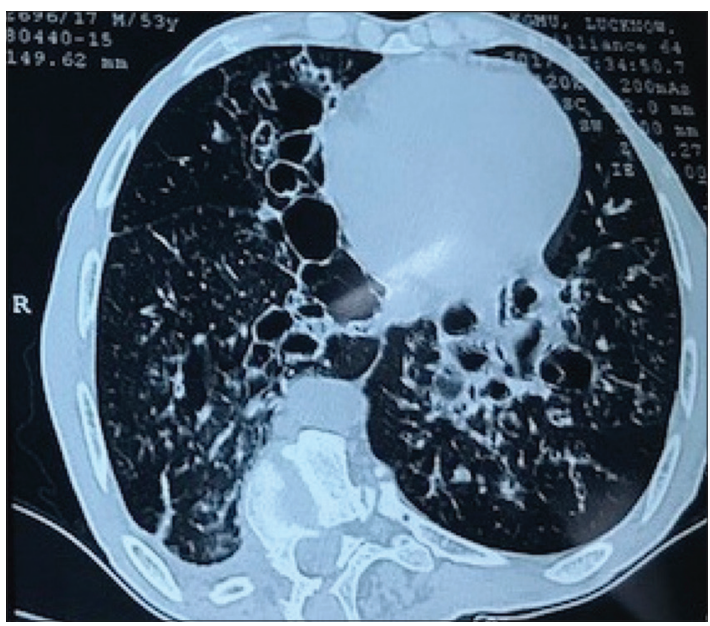

Figure 3: High-resolution computed tomography thorax with contrast revealing bilateral cystic bronchiectasis
[Figure 2]. High-resolution computed tomography (HRCT) of the thorax with contrast revealed bilateral cystic bronchiectasis in both the lungs (bilateral apical and posterior segments of the lower lobe) and a heterogeneously enhancing mass lesion in the right lung posteriorly from pleural origin. A heterogeneous intrathoracic mass lesion on the left side abutting the arch of the aorta was also seen [Figures 3 and 4]. CT-guided biopsy from both the mass lesions showed cores of fibrocollagenous tissue, with tumor cells arranged in sheets and fascicles. The tumor cells were bipolar, spindle shaped with fibrillary cytoplasm and elongated wavy spindle-shaped nuclei. The tumor cells were found to be positive for S-100 and vimentin on immunohistochemistry, suggesting neurofibroma [Figure 5]. Based on clinical, radiological, and histopathological examination findings, a diagnosis of bilateral bronchiectasis and pulmonary neurofibroma was made. The patient was started on intravenous antibiotic infusion of piperacillin-tazobactam $4.5 \mathrm{~g}$ QID and oral azithromycin $500 \mathrm{mg}$ OD for 7 days. His fever started regressing from day 2 , and he was afebrile thereafter. The patient was continued for

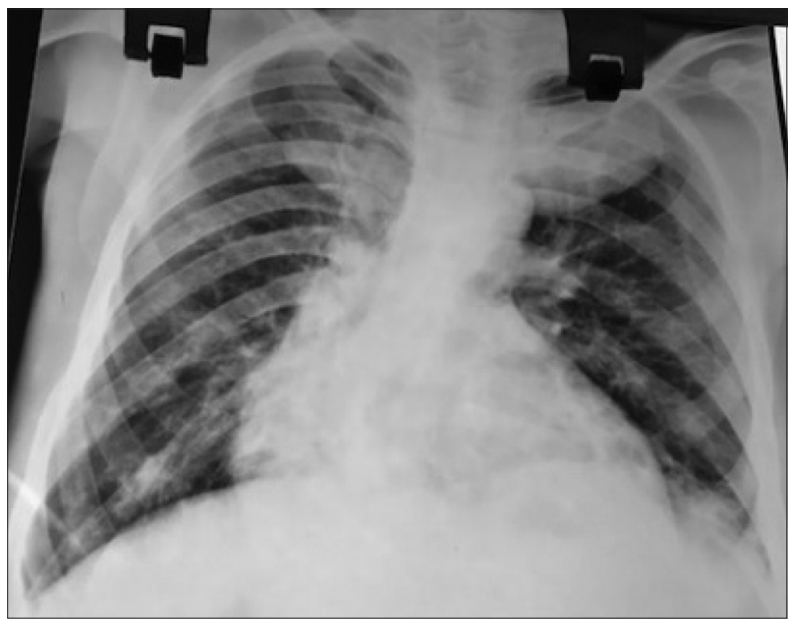

Figure 2: Chest X-ray posteroanterior view showing heterogeneous opacity in the left upper zone and right paracardiac region

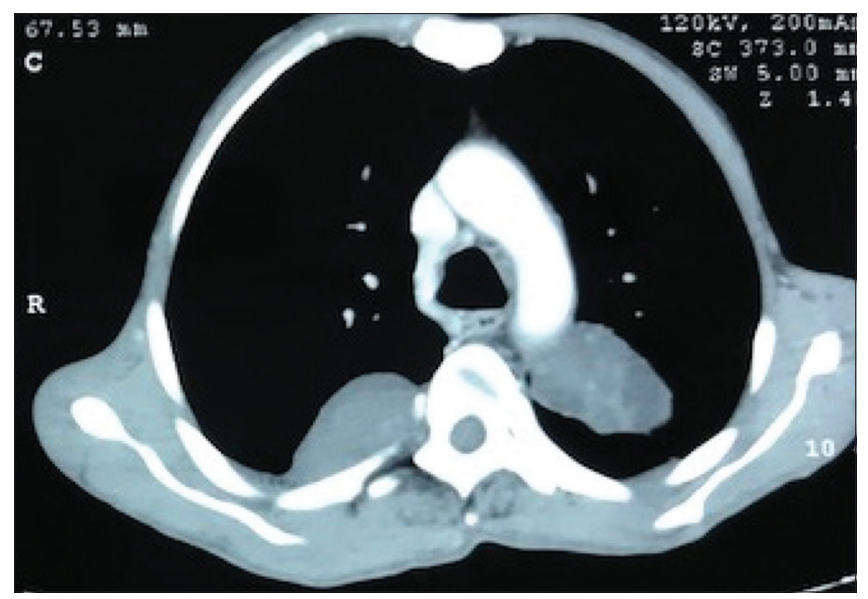

Figure 4: Mediastinal window on computed tomography thorax showing a heterogeneously enhancing mass lesion in the posterior mediastinum 
1 week with the in-hospital antibiotic regimen. At discharge, the patient was prescribed oral azithromycin in a single daily dose for the next 5 days. The patient was relieved of his symptoms (cough and fever). He was referred to thoracic surgery for removal of the intrathoracic neurofibroma.

\section{Discussion}

NF1 (von Recklinghausen's disease) is a genetic disease with involvement of multiple organs of neuroectodermal origin. This group of diseases is also known as phakomatoses, from the Greek 'phakos' meaning 'stigmatized at birth'. ${ }^{[6]}$ Neurofibromatosis is a benign tumor which consists of neurilemma cells and fibroblasts. The main symptoms concern skin, nervous system, bones, and eyes. The gene responsible for the development of NF1, known as NF1, is located on the long arm of the $17^{\text {th }}$ chromosome, and encodes a protein known as neurofibromin. ${ }^{[7]}$ Thoracic manifestations of NF1 are varied and common such as chest wall neurofibroma, kyphoscoliosis, and ribbon-like rib deformity. However, the involvement of lung parenchyma in neurofibromatosis appears to be uncommon. ${ }^{[8]}$ Inactivation of the NF1 gene leads to a permanent stimulation of a cascade of signals and excessive cell division, leading to the formation of tumors. It can develop anywhere on the peripheral nerve, and can be seen most frequently on the trunk, lower extremity, head, and upper extremity, but less in the mediastinum and perineum. Respiratory neurofibromatosis is very uncommon. ${ }^{[9]}$

Neurogenic tumors of the lung originate from the various nervous structures of the costovertebral recesses and less frequently from the pleuro-pulmonary nerves. Schwannoma (neurilemmoma) and neurofibroma are the most common mediastinal neurogenic tumors. Both these lesions are benign, slow-growing tumors. Most commonly, they appear as encapsulated and well-marginated masses found in the costovertebral sulci where they arise from the intercostal nerve rami. In the case of lung involvement, it can cause obstruction, chest deformities, airway and parenchymal neurogenic tumors, pulmonary fibrosis, cystic pulmonary diseases, primary pulmonary hypertension, central hypoventilation, and diaphragm paralysis.

Over one-third of patients with neurofibromas will have neurofibromatosis (von Recklinghausen's disease). These patients tend to present at an earlier age and may have café-au-lait spots, suggesting the diagnosis. Pleural neurofibromatosis arises from the intercostal nerve, manifests as chest distress and dyspnea on exertion. Langman et al. reported two cases of pleural neurofibroma. ${ }^{[10]}$ Surgical resection is the definitive treatment for these benign nerve sheath tumors, commonly performed through video-assisted thoracoscopic surgery with favorable results. Surgery for mediastinal mass, which has been diagnosed as neurofibroma is done only if it causes compression or quite large. ${ }^{[11]}$

A literature search revealed that the coexistence of bronchiectasis and pulmonary neurofibroma is rarely seen in neurofibromatosis. However, sporadic case reports of pulmonary manifestations due to neurofibromatosis do exist. The first published article of neurofibromatosis-induced pulmonary manifestations was in 1963 when Davies reported a case of neurofibromatosis and interstitial lung disease. ${ }^{[12]}$ The exact prevalence and reason for its association is still unclear.

Zamora et al.'s case series and literature review based on CT morphology combined with extra-pulmonary manifestation suggested that neurofibromatosis with diffuse lung disease is a distinct clinical entity, characterized by upper-lobe cystic, bullous disease, emphysema, basilar fibrosis, and ground-glass opacity. HRCT findings revealed emphysema (25\%), cyst (25\%), ground-glass abnormality (37\%), bullae (50\%), and reticular abnormalities $(50 \%)$ in patients with neurofibromatosis [Table 1]..$^{[4]}$

Bronchiectasis is defined as abnormal, persistent bronchial dilatation, usually associated with inflammation in the bronchial tree and lung parenchyma. The close differential diagnosis for this could be cystic fibrosis, allergic bronchopulmonary aspergillosis, nontubercular mycobacteria

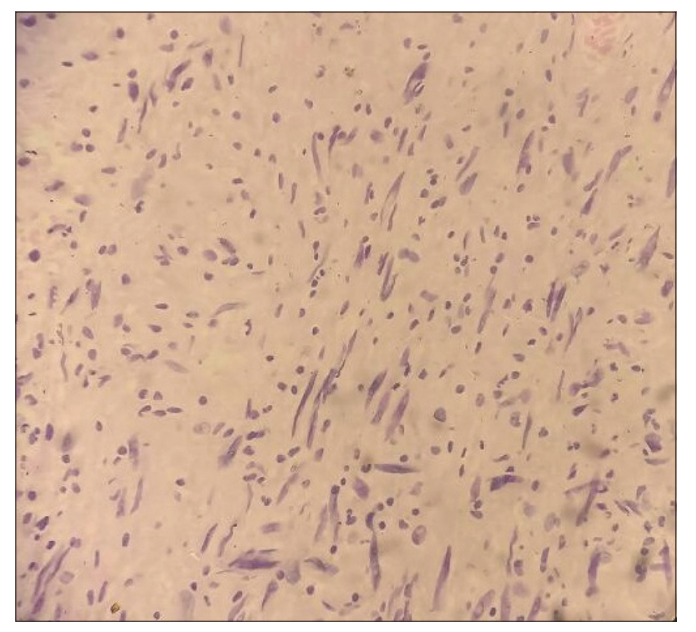

Figure 5: Intrathoracic mass biopsy histopathological examination showing cores of fibrocollageneous tissue with a tumor arranged in sheets and fascicle. The tumor cells are bipolar, spindle shaped with fibrillary cytoplasm and elongated wavy spindle-shaped nuclei

\begin{tabular}{lc}
\hline $\begin{array}{l}\text { Table 1: Prevalence of different lung diseases in } \\
\text { neurofibromatosis }{ }^{[4,14]}\end{array}$ \\
\hline Lung pathology & Percentage \\
\hline Cyst & $14-25$ \\
Bullae & $14-25$ \\
Emphysema & $18-50$ \\
Reticular & 50 \\
Ground-glass opacity & 37 \\
Honeycombing & 13 \\
Benign tumors (neurofibroma) & 11.3 \\
Primary lung carcinoma & 1.1 \\
Malignant nerve sheath tumor & 2.2 \\
Lateral meningocele & 2.2 \\
\hline
\end{tabular}


Pandey, et al.: Pulmonary neurofibromatosis with bronchiectasis

\begin{tabular}{ll}
\hline \multicolumn{2}{l}{ Table 2: Differences between cyst and bronchiectasis } \\
\hline Cyst & Bronchiectasis \\
\hline Air-filled, round parenchymal spaces & $\begin{array}{l}\text { Dilated bronchi, distortions of normal bronchial shape, such as varicoid (string of pearls) or } \\
\text { cystic (cluster of grapes sign) morphology }\end{array}$ \\
Low attenuating area & $\begin{array}{l}\text { Bronchial thickening, mucus plugging can be seen } \\
\text { Increased bronchoarterial ratio, lack of bronchial tapering }\end{array}$ \\
Thin wall $<2 \mathrm{~mm}$ & $\begin{array}{l}\text { Signet ring sign, corresponding to a dilated bronchus immediately adjacent to a smaller } \\
\text { companion pulmonary artery }\end{array}$ \\
Contain air, sometime air plus fluid & $\begin{array}{l}\text { Causes are postinfection, allergic bronchopulmonary aspergillosis, cystic fibrosis, } \\
\text { nontubercular mycobacteria, common variable immunodeficiency, etc. }\end{array}$ \\
Causes LAM-round, smooth cyst, diffuse distribution & \\
LIP - Random, perivascular cyst, diffuse distribution & \\
PLCH - Bizarre irregular cyst upper-lobe predominance & \\
BHDS - Round, lentiform cyst, basilar subpleural distribution & \\
\hline LAM: Lymphangioleiomyomatosis, LIP: Lymphocytic interstitial pneumonia, PLCH: Pulmonary Langerhans cell histiocytosis, BHDS: Birt-Hogg-Dubé \\
syndrome
\end{tabular}

infection, primary ciliary dysfunction, common variable immunodeficiency, postinfection, etc. ${ }^{[13]}$ Pulmonary cyst is an air-filled lucency or low-attenuating area bordered by a thin wall (usually $<2 \mathrm{~mm}$ ) and having a well-defined interface with normal lung tissue. ${ }^{[14]}$ The differences between bronchiectasis and lung cysts are given in Table 2.

However, our patient had predominantly lower lobe-involvement bronchiectasis. In most of the case series and observational studies of neurofibromatosis, the pulmonary cyst was seen in the upper lobe of the lung. However, bronchiectasis has not been described. ${ }^{[15]}$ This case is unique because our patient presented with bilateral cystic bronchiectasis, kyphoscoliosis, and multiple skin neurofibromas with pulmonary neurofibroma.

\section{ConcLusion}

Patients with neurofibromatosis and respiratory symptoms need to be checked for possible changes in the lung parenchyma, and they require a multidisciplinary approach. A few studies showed upper and lobe cystic and bullous disease and basilar fibrosis unlike our patient who had predominantly lower-lobe bronchiectasis with bilateral pulmonary neurofibroma as a phenomenal thoracic presentation of neurofibromatosis.

\section{Declaration of patient consent}

The authors have certified that they have obtained all appropriate patient consent forms. In the form, the patient has given his consent for his images and other clinical information to be reported in the journal. The patient understands that his name and initial will not be published, and due efforts will be made to conceal his identity, but anonymity cannot be guaranteed.

\section{Financial support and sponsorship}

Nil.

\section{Conflicts of interest}

There are no conflicts of interest.

\section{REFERENCES}

1. Lammert M, Friedman JM, Kluwe L, Mautner VF. Prevalence of neurofibromatosis 1 in German children at elementary school enrollment. Arch Dermatol 2005;141:71-4.

2. Huson SM. The neurofibromatosis: Classification, clinical features and genetic counselling. In: Kaufmann D, editor. Neurofibromatoses. Monogr Hum Genet. Manchester (UK): Basel \& Karger; 2008; p. 1-20.

3. Nardecchia E, Perfetti L, Castiglioni M, Di Natale D, Imperatori A, Rotolo N. Bullous lung disease and neurofibromatosis type-1. Monaldi Arch Chest Dis 2012;77:105-7.

4. Zamora AC, Collard HR, Wolters PJ, Webb WR, King TE. Neurofibromatosis-associated lung disease: A case series and literature review. Eur Respir J 2007;29:210-4.

5. Ribet ME, Cardot GR. Neurogenic tumors of the thorax. Ann Thorac Surg 1994;58:1091-5.

6. Itoi K, Yanagihara K, Okubo K, Kuwabara M, "A case of lung cancer in a patient with von Recklinghausen's disease." Nihon Kyobu Shikkan Gakkai Zasshi 1992;30:317-21.

7. Shimizu Y, Tsuchiya S, Watanabe S, Saitoh R. von Recklinghausen's disease with lung cancer derived from the wall of emphysematous bullae. Intern Med 1994;33:167-71.

8. De Scheerder I, Elinck W, Van Renterghem D, Cuvelier C, Tasson J, Van der Straeten M. Desquamative interstitial pneumonia and scar cancer of the lung complicating generalised neurofibromatosis. Eur J Respir Dis 1984;65:623-6.

9. Yuan T, Luo BL, Gu QH, Yao J. Analysis of two cases with bronchopulmonary neurofibromatosis. Multidiscip Respir Med 2012;7:17

10. Langman G, Rathinam S, Papadaki L. Primary localised pleural neurofibroma: expanding the spectrum of spindle cell tumours of the pleura. J Clin Pathol 2010;63:116-8.

11. Cardillo G, Carleo F, Khalil MW, Carbone L, Treggiari S, Salvadori L, et al. Surgical treatment of benign neurogenic tumours of the mediastinum: A single institution report. Eur J Cardiothorac Surg 2008;34:1210-4.

12. Davies PB. Diffuse pulmonary involvement in von Recklinghausen's disease: A new syndrome. Thorax 1963;18:198.

13. Polverino E, Goeminne PC, McDonnell MJ, Aliberti S, Marshall SE, Loebinger MR, et al. European respiratory society guidelines for the management of adult bronchiectasis. Eur Respir J 2017;50: 1700629.

14. Grant LA, Babar J, Griffin N. Cysts, cavities, and honeycombing in multisystem disorders: Differential diagnosis and findings on thin-section CT. Clin Radiol 2009;64:439-48.

15. Ueda K, Honda O, Satoh Y, Kawai M, Gyobu T, Kanazawa T, et al. Computed tomography (CT) findings in 88 neurofibromatosis 1 (NF1) patients: Prevalence rates and correlations of thoracic findings. Eur J Radiol 2015;84:1191-5. 